\title{
Holographic image processing of three-dimensional objects
}

\author{
Jonathan Maycock,${ }^{a}$ Conor P. Mc Elhinney, ${ }^{a}$ Alison E. Shortt, ${ }^{a}$ Thomas J. Naughton, ${ }^{a}$ John \\ B. McDonald, ${ }^{a}$ Brian M. Hennelly, ${ }^{b}$ Unnikrishnan Gopinathan,${ }^{b}$ David S. Monaghan, ${ }^{b}$ John T. \\ Sheridan, ${ }^{b}$ and Bahram Javidi $^{c}$ \\ ${ }^{a}$ Department of Computer Science, National University of Ireland, Maynooth, County Kildare, \\ Ireland \\ ${ }^{b}$ Department of Electronic and Electrical Engineering, National University of Ireland, Dublin, \\ Ireland \\ ${ }^{c}$ Electrical and Computer Engineering, University of Connecticut, 371 Fairfield Road, Unit \\ 1157 Storrs, CT 06269, USA
}

\begin{abstract}
We report on recent advances made in the area of holographic image processing of three-dimensional (3D) objects. In particular, we look at developments made in the areas of encryption, compression, noise removal, and 3D shape extraction. Results are provided using simulated objects and real-world 3D objects captured using phase-shift digital holography.
\end{abstract}

Keywords: three-dimensional image processing, digital holography, encryption, compression, independent component analysis, 3D shape measurement

\section{INTRODUCTION}

Digital holography ${ }^{1-6}$ has recently become feasible due to advances in megapixel charge coupled device (CCD) sensors with high spatial resolution and high dynamic range. The advantage of digital holography over traditional holography is that the resulting hologram can easily be stored electronically, or, transmitted over conventional communication channels. This paper looks at recent developments made by our group, in the areas of encryption, compression, noise removal using Independent Component Analysis (ICA), and a depth-from-defocus technique, applied to digital holograms of real world objects.

Optical encryption, ${ }^{7-18}$ often produces a complex-valued encrypted image resulting from a random phase mask positioned in the input, Fresnel, or Fraunhofer domain, or combination of domains. Digital holography has been applied to the encryption of $2 \mathrm{D}$ conventional (real-valued) images. ${ }^{13-15}$ Of these, the techniques based on phase-shift interferometry ${ }^{3,5,19}$ (PSI) make good use of detector resources in that they capture on-axis encrypted digital holograms. ${ }^{14,15}$ The PSI technique has also been extended to the encryption of $3 \mathrm{D}$ objects. ${ }^{16}$ In this paper, we consider optical systems that encrypt 2D and 3D objects. Our 3D objects are captured using in-line phase-shift digital interferometry. ${ }^{5,6,20}$ In addition to amplitude, optical systems offer many degrees of freedom to encode data such as phase, ${ }^{8,10,17,21-23}$ polarization, ${ }^{24}$ and wavelength. ${ }^{11}$ In our system, the encryption is performed using a random phase mask in a Fresnel plane. The random phase mask, acting as the encryption/decryption key, is displayed on a Spatial Light Modulator (SLM). The large space bandwidth product of the SLM means that key sizes in the order of hundreds of thousands or even millions of digits are feasible. The encrypted data captured by the CCD camera is a complex-valued white-noise-like signal. When decrypting, the complex-valued data (or some real function of it) is displayed on the first SLM and the decoding mask is displayed on the second SLM. We use a virtual optics method to simulate the encryption and decryption process. The efficient linear canonical transform (LCT) algorithm, proposed by Hennelly and Sheridan, ${ }^{25}$ can simulate any such optical system. The display of the encrypted image and decoding mask on SLMs results in quantization which is dependent on the number of discrete amplitude or phase levels that are offered by each

Further author information: JM: jmaycock@cs.may.ie; JMcD: johnmcd@cs.may.ie; TN: tom.naughton@may.ie; JS: John.Sheridan@ucd.ie; BJ: bahram@engr.uconn.edu 
SLM. After encryption, the size (in terms of memory for storage and transmission) of the digital holograms can be still quite large. Therefore, compression of encrypted holograms was also investigated.

Quantization in holograms, ${ }^{26,27}$ and compression of real-valued ${ }^{28}$ and complex-valued ${ }^{23,29-31}$ digital holograms has received some attention to date. Some studies have also been performed on the decrypted-domain effects of perturbations, including quantization, in the encrypted domain. ${ }^{32,33}$ When gauging the errors introduced by lossy compression, we are not directly interested in the defects in the hologram itself, only how compression noise affects the quality of reconstructions of the compressed 3-D object. Our holograms were encrypted by perturbing the Fresnel diffraction of the 3-D objects with a random phase mask. We simulated this encryption step in software. ${ }^{34}$ The dimensions of each encrypted hologram are $1024 \times 1024$ pixels. Encrypted digital holograms have been successfully quantized previously. We extend these results ${ }^{23}$ by choosing nonuniform distributions of quantization values. We consider each complex-value as a vector of length two and use an unsupervised artificial neural network (ANN) to locate the most suitable clusters in the encrypted digital hologram data. We then quantize our encrypted holograms with the centers of these clusters. We use a reconstructed-object-plane NRMS metric to quantify the quality of our decompressed and decrypted holograms. Next, we looked at a technique which attempts to reduce speckle noise in digital holograms.

Any imaging system that uses coherent light will have its images corrupted by speckle noise. ${ }^{35}$ This speckle becomes an important drawback in the application of image processing techniques to digital holographic data. We have investigated the use of ICA for the removal of speckle noise from digital holograms. ICA is a statistical method for transforming an observed multidimensional random vector into components that are statistically as independent from each other as possible. It can be seen as an extension of Principal Component Analysis (PCA) and factor analysis. ${ }^{36}$ ICA is a much more powerful technique, however, capable of finding the underlying factors or sources when these classic methods fail completely. Typical examples where ICA has been applied successfully for the analysis of multivariate data range from brain waves recorded by multiple sensors to interfering radio signals arriving at a mobile phone. ${ }^{37}$ We pose the issue of noise removal in our data as a Blind Source Separation problem. Specifically we treat scene reconstructions as observation data formed through the combination of two sources. The first source is due to the scene itself, and the second source is due to the noise. By applying ICA to a collection of these observations, a set of resulting sources is identified, one of which is the scene data, and the other is the noise. For our experiments speckle is considered to be multiplicative noise.

Finally, we present a technique that converts a digital hologram into a surface in 3D space. Many existing 3D imaging techniques are based on the explicit combination of several two-dimensional perspectives through digital image processing. Multiple perspectives of a 3D object can be combined optically, in parallel, and stored together as a single complex-valued digital hologram. We apply a technique called depth-from-defocus to our digital holograms to create depth maps of the objects encoded in our holograms. Several approaches for applying depth-from-defocus measures to holograms have been reported in literature. However the majority of these techniques simply identify the optimal focal distance for the entire hologram. For example the technique's of Liebling and Unser, ${ }^{38}$ and that of Gillespie and King, ${ }^{39}$ were used to autofocus the full complex wave field and return the point where it was most accurately focused. Ma et al. ${ }^{40}$ used variance to calculate a 3D reconstruction image from a digitized analog hologram. In this paper we apply variance to recover the 3D depth information from holograms captured using PSI.

\subsection{Outline of the paper}

In Sect. 2 we explain our encryption and decryption mechanism, and in Sect. 2.1 explain our method for measuring quantization tolerances, and detail results of our investigations into SLM quantization. We then apply a lossy technique of quantization to the real and imaginary encrypted components of each holographic pixel in Sect. 3. Section. 4 details an experiment whereby ICA was used to remove speckle noise from digital holograms. In Sect. 5, a technique to convert a digital hologram into a cloud of surface points in 3D space is presented. Finally, conclusions are drawn in Sect. 6.

\section{ENCRYPTION AND DECRYPTION}

In this section, we describe the process of encryption and decryption of the data. The following analysis is carried out in one dimension with the extension to two dimensions being straightforward. Let $f(x)$ represent the 


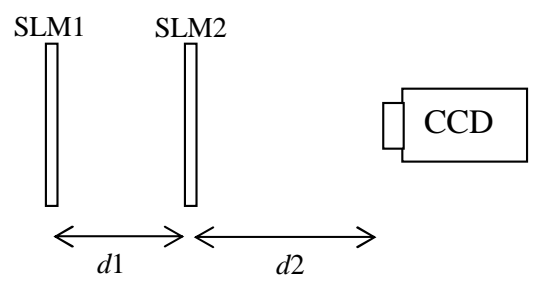

(a)

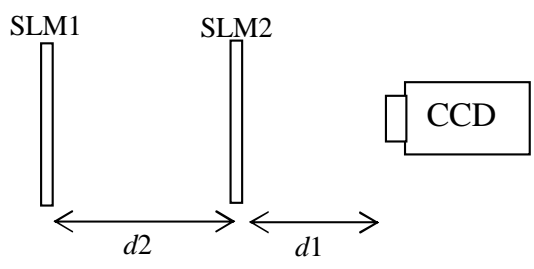

(b)

Figure 1. Schematic of the optical system for encryption and decryption. (a) SLM1 and SLM2 are used to display the input image and encryption key, respectively, and (b) SLM1 and SLM2 are used to display the encrypted image and the decrypted key, respectively.

input data to be encrypted. Let $L_{\alpha 1, \beta 1, \gamma 1}$ and $L_{\alpha 2, \beta 2, \gamma 2}$ represent LCTs corresponding to two combinations of lenses and free space propagation distances. The input signal $f(\cdot)$ is transformed using $L_{\alpha 1, \beta 1, \gamma 1}$, multiplied by a random phase mask $R$ and transformed again using $L_{\alpha 2, \beta 2, \gamma 2}$.

The LCT $L_{\alpha, \beta, \gamma}$ of $f(\cdot)$ is a three parameter transform described as

$$
L_{\alpha, \beta, \gamma}[f]\left(x^{\prime}\right)=K \iint f(x) \exp \left[j \pi\left(\alpha x^{2}-2 \beta x x^{\prime}+\gamma x^{\prime 2}\right)\right] \mathrm{d} x .
$$

The encrypted signal $\Psi(\cdot)$ can be written as

$$
\Psi(\cdot)=L_{\alpha 2, \beta 2, \gamma 2}\left[L_{\alpha 1, \beta 1, \gamma 1}(f) R\right]
$$

and can be decrypted with

$$
f(\cdot)=L_{-\alpha 1,-\beta 1,-\gamma 1}\left\{R^{*} L_{-\alpha 2,-\beta 2,-\gamma 2}[\Psi(\cdot)]\right\},
$$

where $L_{-\alpha,-\beta,-\gamma}$ is the inverse transform of $L_{\alpha, \beta, \gamma}$ and $*$ indicates complex conjugate. There is a second method of decryption, suitable for optical implementation, using the following property of the LCT

$$
L_{-\alpha,-\beta,-\gamma}(f)=\left[L_{\alpha, \beta, \gamma}\left(f^{*}\right)\right]^{*} .
$$

Using this property, we can write Eq. 3 as

$$
f^{*}(\cdot)=L_{\alpha 1, \beta 1, \gamma 1}\left\{R L_{\alpha 2, \beta 2, \gamma 2}\left[\Psi^{*}(\cdot)\right]\right\} .
$$

If $f(\cdot)$ is real, $f^{*}(\cdot)=f(\cdot)$. If one takes the conjugate of the encrypted signal and does the LCT operations used for encryption in reverse order, one gets the conjugate of the input signal. In the present work, $L_{\alpha 1, \beta 1, \gamma 1}$ and $L_{\alpha 2, \beta 2, \gamma 2}$ corresponds to free space distances $d_{1}$ and $d_{2}$ in which case

$$
\alpha 1=\beta 1=\gamma 1=\frac{\pi}{\lambda d_{1}}, \alpha 2=\beta 2=\gamma 2=\frac{\pi}{\lambda d_{2}}
$$

where $\lambda$ is the wavelength of light used.

In our experiments, we used digitized photographs as our $2 \mathrm{D}$ objects. The phase mask consisted of values chosen with uniform probability from the range $[0,2 \pi)$ using a pseudo-random number generator. Digital holograms of 3D objects were captured using an in-line PSI set-up ${ }^{6,20}$ based on a Mach-Zehnder interferometer. Encrypted versions of these holograms can be obtained by positioning the phase mask between the object and the digital camera. For our experiments, this encryption step was simulated ${ }^{23}$ after optical capture of the real-world 3D objects.

\subsection{Quantization Errors due to SLM}

Figure 1 shows the optical system used for encryption and decryption. In Fig. 1(a), SLM1 and SLM2 are used to display the input image and encryption key, respectively. In Fig. 1(b), SLM1 and SLM2 are used to display 


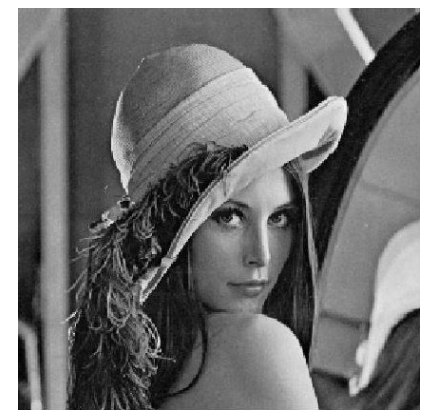

(a)

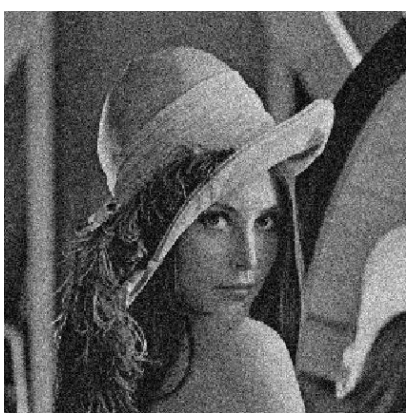

(c)

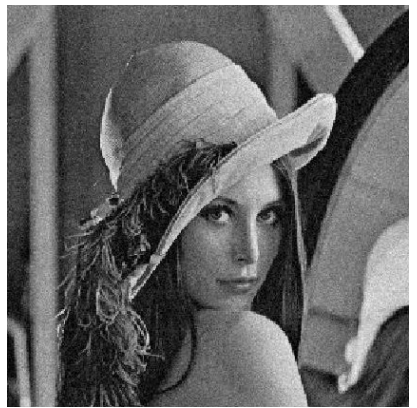

(b)

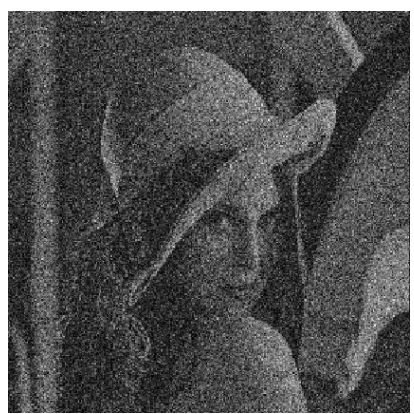

(d)

Figure 2. The decrypted 2D image with (a) no quantization, and decrypted with (b) 64, (c) 8, and (d) 2 quantization levels in the phase mask.

the encrypted image and the decryption key, respectively. We perform the encryption and decryption process by a virtual optics method using the discrete Fresnel transform, which is a special case of the discrete LCT and for which efficient $n \log (n)$ time algorithms exist. ${ }^{25}$ The wavelength of the coherent source was $532 \mathrm{~nm}$, the distances $d_{1}$ and $d_{2}$ were $80 \mathrm{~mm}$, the CCD pixel size was $9 \mu \mathrm{m}$ and the SLM pixel size was $36 \mu \mathrm{m}$. We assumed a $100 \%$ fill factor for both the SLM and CCD.

The error in the decoded image when the SLM is used to represent the encrypted image is studied by modifying the encrypted image according to

$$
\Psi_{i}^{\prime}=\Psi_{i}+\Delta \Psi_{i}
$$

where $\Psi_{i}$ represents the value of the $i$ th pixel in the encrypted image, $\Psi_{i}^{\prime}$ represents the corresponding value represented by the SLM, and $\Delta \Psi_{i}$ is the resulting quantisation error for that pixel. If $a_{i}$ represents the amplitude and $\phi_{i}$ represents the phase of each quantised complex value $\Psi_{i}^{\prime}$ then $a_{i} \in\left\{a^{1} \ldots a^{n}\right\}$ for $n$ predefined amplitude levels and $\phi_{i} \in\left\{\phi^{1} \ldots \phi^{n}\right\}$ for $n$ predefined phase levels. The error $r$ in the decoded image is calculated using a normalised rms (NRMS) error metric defined as

$$
r=\left(\sum_{i=1}^{N} \sum_{j=1}^{N}\left|I_{\mathrm{d}}(i, j)-I(i, j)\right|^{2}\right)^{\frac{1}{2}}\left(\sum_{i=1}^{N} \sum_{j=1}^{N}|I(i, j)|^{2}\right)^{-\frac{1}{2}}
$$

where $I_{\mathrm{d}}(\cdot)$ and $I(\cdot)$ are the intensities of the decrypted and original images, respectively.

\subsection{Phase Quantization}

In this section we present some results from our simulation study. Figure 2 (a) shows the $512 \times 512$ pixel grayscale $2 \mathrm{D}$ image used in our study. We corrupted the 2D image with random phase to make it correspond to the full Fresnel field immediately in front of a reflective real-world object. The image was encrypted as described in Sect. 2. The encryption phase mask had 256 discrete levels. First we studied the quantization effect of the 


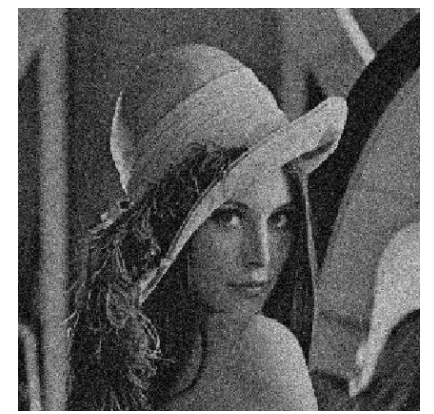

(a)

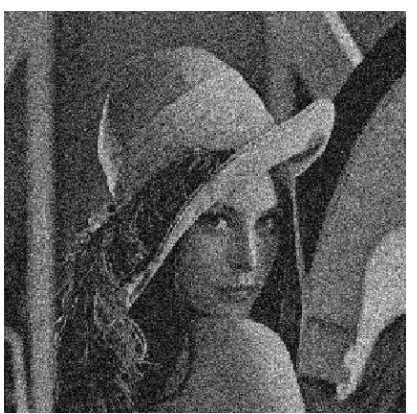

(c)

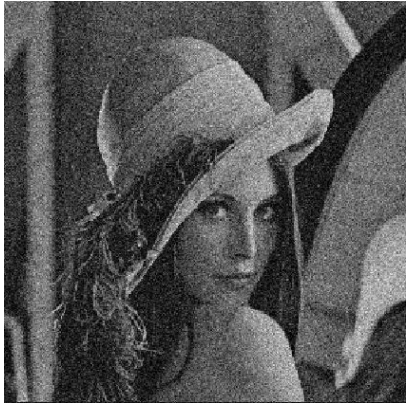

(b)

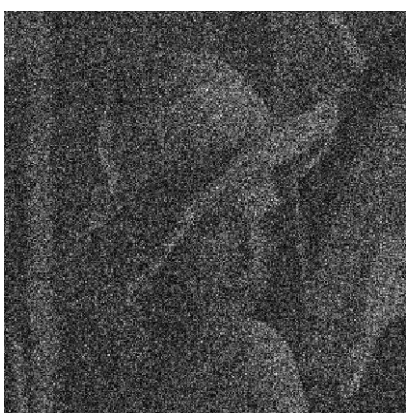

(d)

Figure 3. The decrypted 2D image after the phase-only encrypted image is quantized to (a) 128 , (b) 8 , (c) 4 , and (d) 2 phase levels.

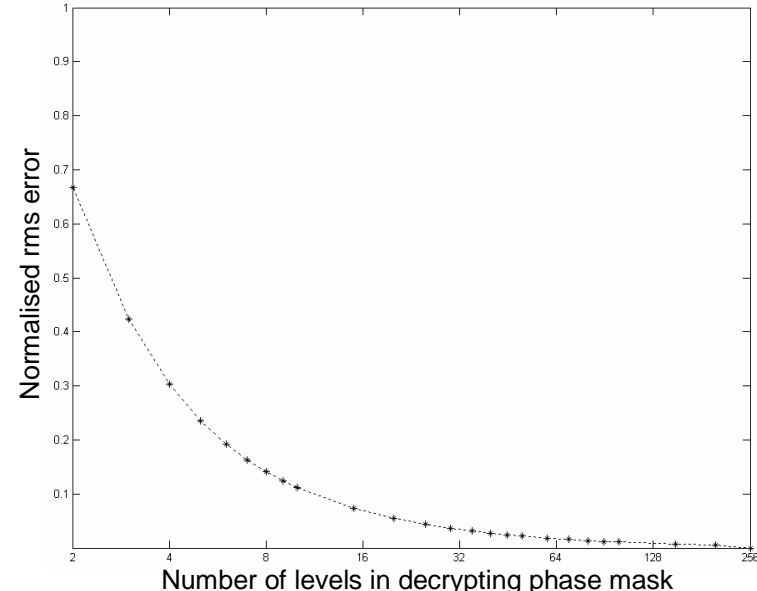

(a)

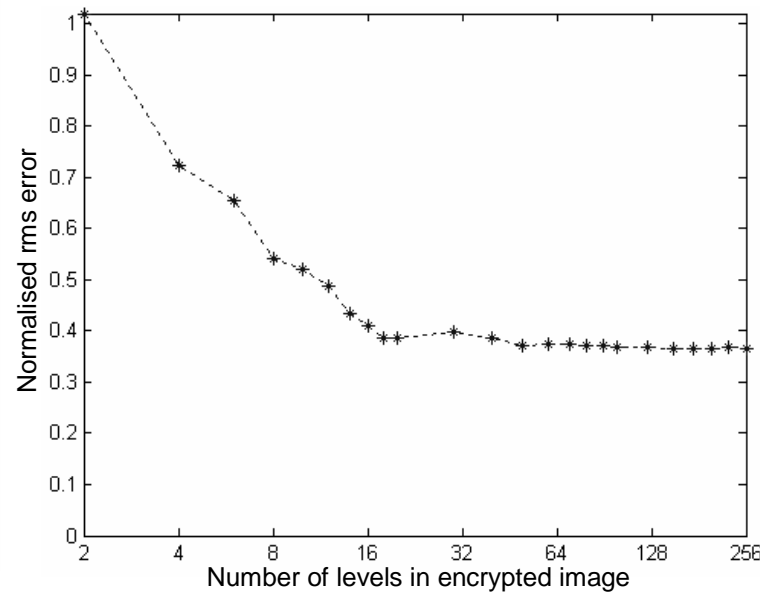

(b)

Figure 4. Plots showing (a) NRMS error as a function of number of quantization levels in the decrypting phase mask, and (b) NRMS error as a function of number of quantization levels in the SLM displaying the phase-only information of the encrypted image. Each plot has a logarithmic scale in its horizontal axis.

phase-only SLM displaying the decoding phase mask. The phase of the decrypting phase mask is quantized to different numbers of discrete levels. Figures 2(b) through $(\mathrm{d})$ show the decrypted image when the phase mask is quantized to various numbers of levels.

Next, we studied the effects due to the finite number of discrete levels of the SLM displaying the encrypted image. We do not consider the amplitude of the encrypted image, only the phase; we assume that we have access to a phase-only SLM. (This amplitude removal or amplitude equalization concept has been shown to be a useful low-cost technique for the reconstruction of digital holograms using phase-only SLMs. ${ }^{30}$ ) The phase 


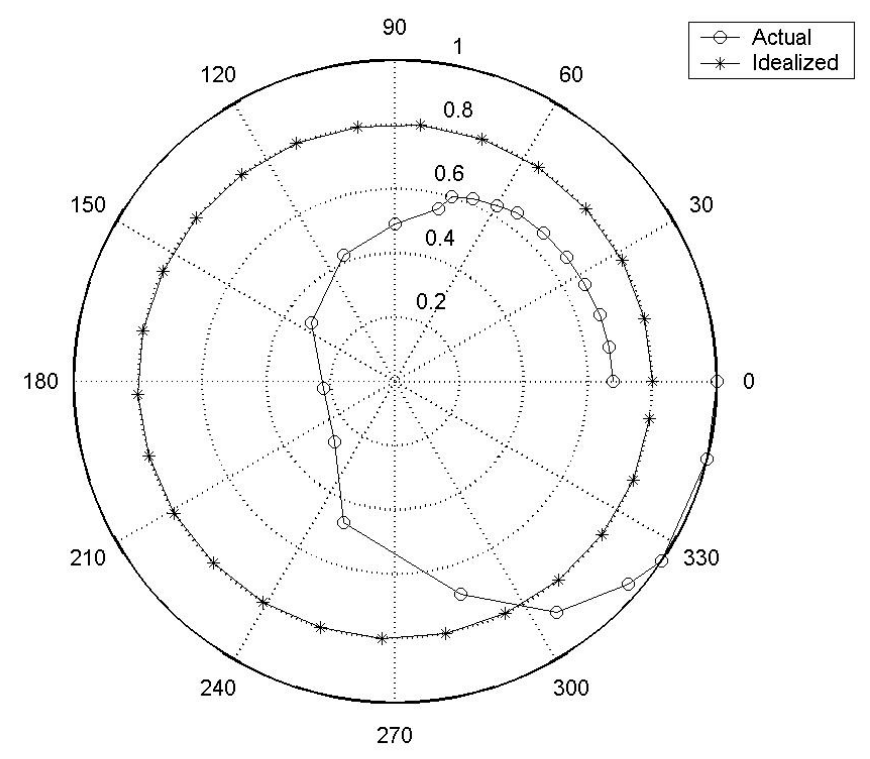

Figure 5. Polar plot of normalized amplitude against phase angle. Plot shows the actual response of the SLM for input grey levels varying from 0 to 255 in quantization steps of 10 levels, and an idealized response with constant amplitude of 0.8 .

of the encrypted image was quantized to different numbers of levels ranging from 2 to 256 . Several of these decrypted images are shown in Fig. 3. Plots of NRMS error as a function of phase levels for each of these two simulation studies are shown in Fig. 4. It was found that the error increases dramatically when the number of phase levels falls below 10 in each case. In Fig. 4(b) the error never falls below approximately 0.4 even when no phase quantization is applied. This has been noticed before in relation to digital holograms ${ }^{30}$ and corresponds to the fundamental losses introduced by amplitude equalization.

\subsection{Error due to Nonlinear Response}

For real-time display of digital holograms we have also proposed a phase-only reconstruction technique. ${ }^{30}$ This technique assumes a linear response SLM. This study is concerned with the non linear response of our SLM. A Mach-Zehnder interferometer was used to characterize our LCD SLM. As is the problem with many electrically addressed SLMs our SLM does not operate in a phase-only mode. Whereas it can produce a phase modulation from 0 to 2 , this is a coupled phase/amplitude modulating mode. Figure 5 shows a polar plot of this undesired amplitude modulation coupled with the phase modulation.

After encryption, compression becomes important in order to reduce the size of our digital holograms, for more efficient storage and transmission purposes.

\section{QUANTIZATION OF ENCRYPTED DIGITAL HOLOGRAMS}

A uniform quantization technique was used to investigate the loss in reconstruction quality due to quantization in encrypted holograms, and to comparatively evaluate the quality of the results obtained using the Kohonen competitive neural network. The uniform quantization technique linearly rescaled the encrypted holograms to the square in the complex plane $[-1-i, 1+i]$ without changing their aspect ratio in the complex plane. The real and imaginary components of each holographic pixel were then quantized. Quantization levels were chosen to be symmetrical about zero; as a result $b$ bits encode $\left(2^{b}-1\right)$ levels. For example, two bits encode levels $\{-1,0,1\}$, three bits encode levels $\{-1,-2 / 3,-1 / 3,0,1 / 3,2 / 3,1\}$, and so on.

Nonuniform quantization was then employed to quantize the encrypted hologram data. A number of techniques were evaluated, but we found that the Kohonen competitive neural network performed best on our digital holograms. It was trained on a $128 \times 128$ pixel window (a subset of the available pixels) of encrypted digital 


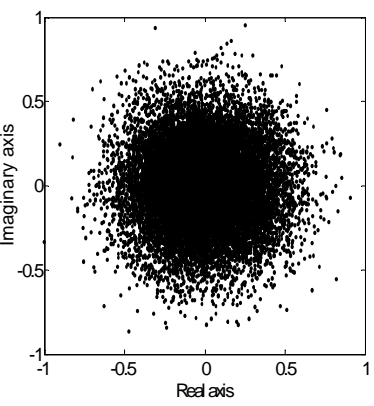

(a)

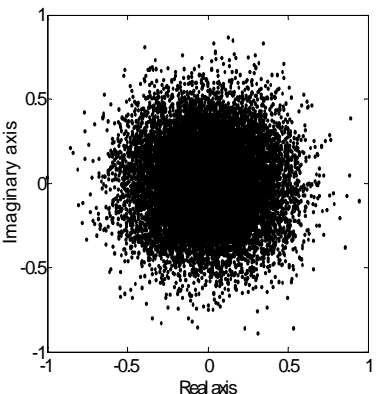

(d)

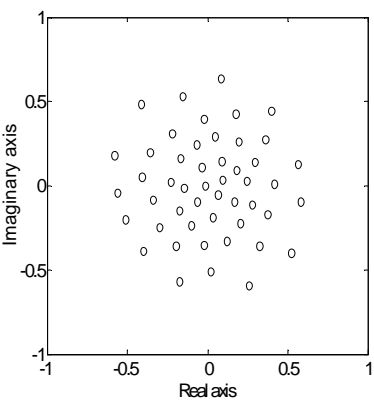

(b)

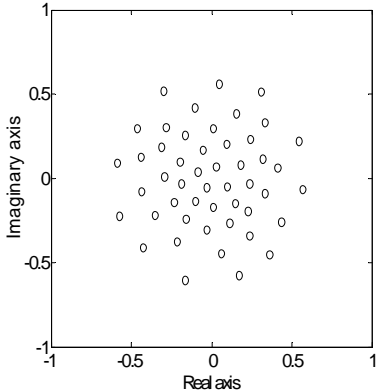

(e)

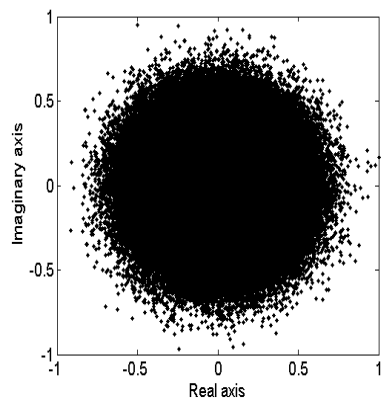

(c)

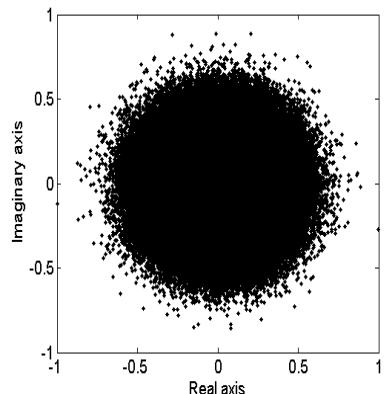

(f)

Figure 6. Scatter plots of the complex-valued encrypted data in the die hologram: (a) $128 \times 128$ window before quantization, (b) $128 \times 128$ window nonuniform quantization (Kohonen competitive), (c) $1024 \times 1024$ window before quantization; and of the data in the bolt hologram: (d) $128 \times 128$ window before quantization, (e) $128 \times 128$ window nonuniform quantization (Kohonen competitive), (f) $1024 \times 1024$ window before quantization

hologram data. We used the resulting centers to quantize a $1024 \times 1024$ pixel encrypted digital hologram. Figure 6 (a) shows a scatter plot of the unquantized $128 \times 128$ pixel window of the die hologram that was used to train the ANN. Figure 6(b) shows the cluster positions found by the ANN (equivalently, this is a scatter plot of the quantized encrypted data). Figure 6 (c) shows a scatter plot of the full $1024 \times 1024$ pixel hologram that the clusters from Fig. 6(b) were applied to. Figures 6(d)-(f) show equivalent scatter plots for the bolt hologram.

Figure 7 shows plots of NRMS difference against number of bits of encrypted holographic data for both uniform quantization and Kohonen competitive quantization. It illustrates the consistently lower NRMS error achieved by Kohonen competitive nonuniform quantization over uniform quantization on our encrypted digital holograms. The reconstructed object intensities for both objects for selected quantization resolutions are shown in Fig. 8. The lower NRMS error, shown in Fig. 7, results in improved quality in the reconstructed objects when using nonuniform quantization compared with uniform quantization. Reductions from 8 bytes to 4 bits, 3 bits, and 2 bits correspond to compression ratios of 16,21 , and 32 , respectively.

Ideally, the cluster centers from one hologram could be stored in a lookup table and applied with reasonable results to the quantization of subsequent holograms. (The JPEG algorithm uses a hard-coded lookup table of cosine-domain quantization values arrived at through performance evaluation over a database of sample input images.) We have found that the set of cluster centers we obtained from the Kohonen competitive neural network is very effective when applied in the quantization of a different hologram. This is illustrated in Fig. 7, where it can be seen that quantizing the die hologram using the centers obtained by applying Kohonen to the bolt hologram results in comparably low NRMS errors compared to those obtained when applying the centers produced specifically for the die hologram. By using the centers obtained from the Kohonen competitive network to quantize other encrypted holograms, we have the improved performance of nonuniform quantization combined with the speed advantage of uniform quantization. 


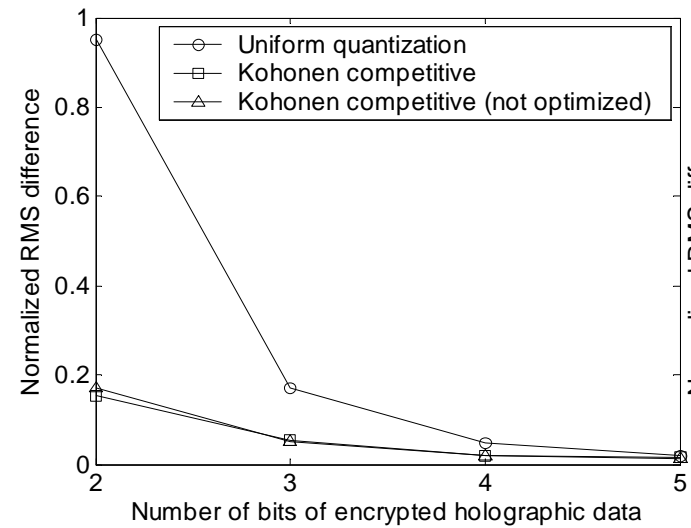

(a)

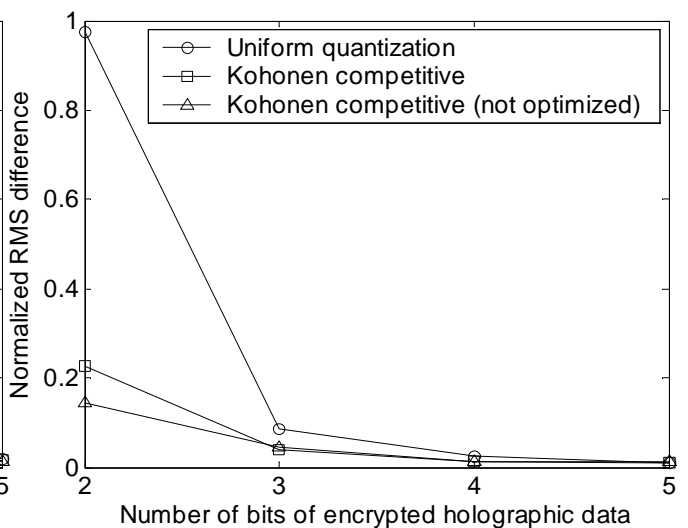

(b)

Figure 7. NRMS intensity difference in the decrypted and reconstructed 3-D objects plotted against quantization level, with uniform quantization and nonuniform quantization (Kohonen competitive): (a) for die hologram, where nonoptimized means using the Kohonen centers from bolt hologram, and (b) for bolt hologram, where non-optimized means using the Kohonen centers from die hologram

\section{INDEPENDENT COMPONENT ANALYSIS APPLIED TO DIGITAL HOLOGRAMS}

Any imaging system that uses coherent light will have its images corrupted by speckle noise. ${ }^{35}$ This speckle becomes an important drawback in the application of image processing techniques to digital holographic data. We have investigated the use of ICA for the removal of speckle noise from digital holograms. ICA can be considered a generalization of the PCA technique, and was first developed rigorously by Comon. ${ }^{41}$ It is one method that can be used to solve the Blind Source Separation problem. Here the observed data is a linear mixture of some source data

$$
X=F Z
$$

where neither the source data, $Z$, nor the mixture parameters, $F$, are known. ICA uses some statistical properties of the source data to estimate the mixture parameters that maximize the contrast between the source components. Once this has been done, an estimate of the source signals follows naturally from Eq. 9.

It turns out that it is enough to assume that the source signals are statistically independent to estimate a set of source signals from their mixtures. Thus ICA can be used to successfully separate source signals from the observed mixtures. ${ }^{37}$

We have applied ICA to the removal of background speckle noise from digital holograms. In our experiments we consider speckle to be a multiplicative noise which can be formulated as $K=I+n I$, where $I$ is an image, and $n$ is a uniformly distributed noise with zero mean and arbitrary variance. In order to bring the multiplicative nature of this noise into an additive, and therefore linear, domain, we took the natural logarithm of the observations before applying standard ICA to them.

\subsection{ICA algorithm}

A common pre-processing step that transforms the data into a suitable form for application of ICA is whitening (sphering). We have used PCA to whiten our data, which results in data vectors that have decorrelated components and are of unit variance. The FastICA ${ }^{42}$ algorithm was then applied to the data. This algorithm estimates a matrix $\mathrm{W}$, which maximizes the negentropy approximation under the constraints that the estimated components are uncorrelated. Given a random vector $y=\left(y_{1}, \ldots, y_{n}\right)^{T}$ the negentropy can be defined as

$$
J(y)=H\left(y_{\text {gauss }}\right)-H(y)
$$




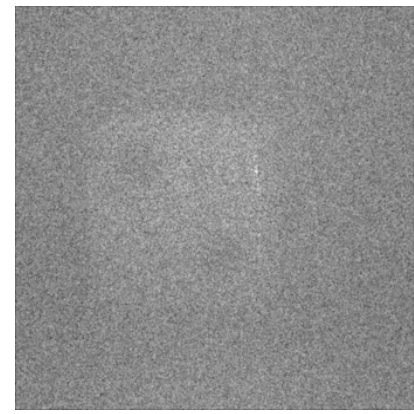

(a)

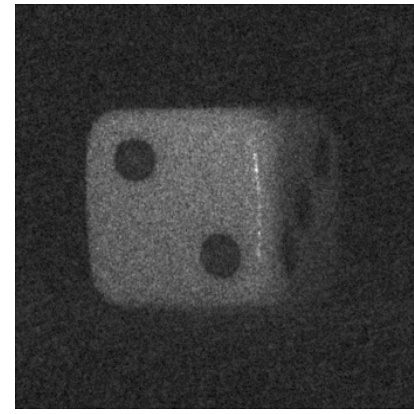

(e)

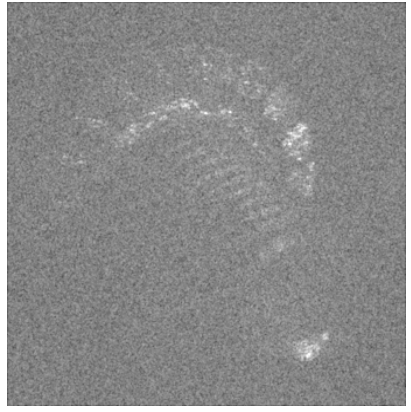

(b)

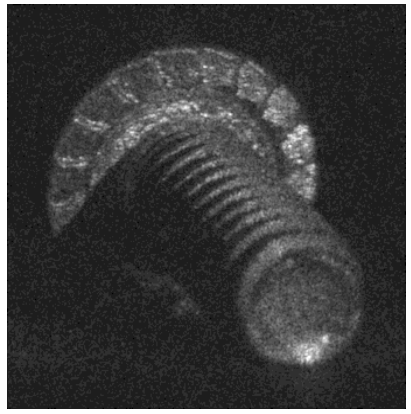

(f)

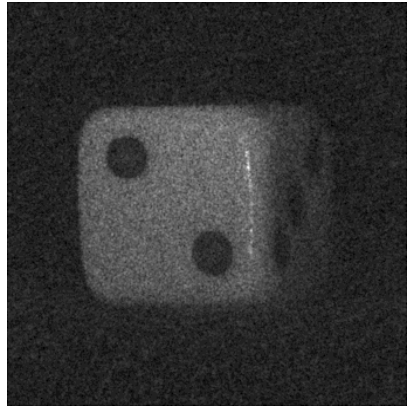

(c)

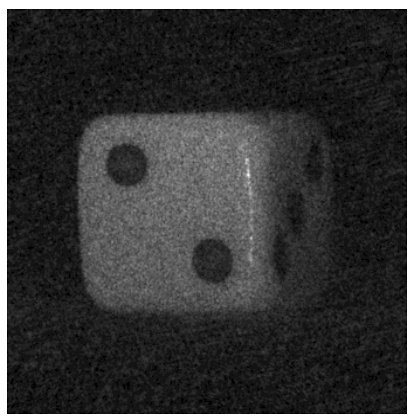

(g)

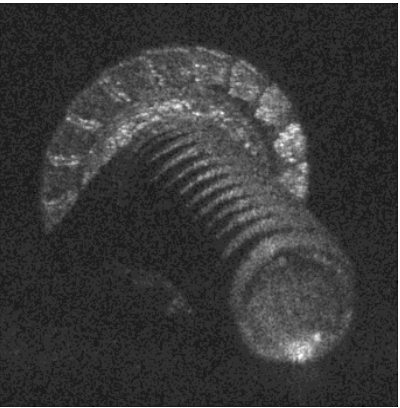

(d)

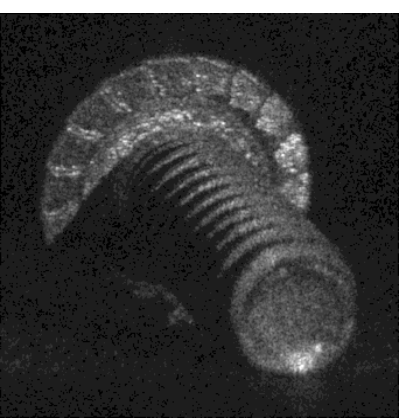

(h)

Figure 8. Reconstructions from encrypted digital hologram data with uniform quantization (upper row) and Kohonen competitive nonuniform quantization (lower row): (a),(b),(e),(f) 2 bits per real and imaginary value, and (c),(d),(g),(h) 3 bits per real and imaginary value. Mean filtering $(5 \times 5$ pixel $)$ was applied in each case

where $y$ gauss is a Gaussian random vector chosen so that it has the same covariance matrix as $y$. The differential entropy $H$ of a random vector $\Im=\left(\Im_{1}, \ldots, \Im_{n}\right)^{T}$ with density $f(\cdot)$ is defined as ${ }^{36}$

$$
H(\Im)=-\int f(\Im) \log f(\Im) d y
$$

As far as statistical properties are concerned, negentropy is the optimal estimator of nongaussianity. ${ }^{43}$ ICA can be seen as the search for directions which are maximally nongaussian. Each local maximum of negentropy gives an estimate of one independent component. ${ }^{37}$ After ICA estimates the source signals, all that remains is to de-whiten the data.

\subsection{Experiments}

For our experiments we used holograms of a bolt and a die. In order to create the two observations needed by ICA, we took two $1024 \times 1024$ pixel windows from the hologram separated in space by one pixel. When these windows were reconstructed, it resulted in a different speckle pattern occurring across the object. The two reconstructions are assumed to be a mixture of speckle noise and object data. The reconstructed windows are first rearranged into 1D vectors. Then their natural logarithm is taken, and as a preprocessing step they are whitened using PCA. This produces decorrelated components of unit variance. Now standard ICA is applied to estimate the original two sources. After ICA has estimated these sources, they are de-whitened. Finally, the 1D vectors are rearranged into a $2 \mathrm{D}$ matrix, and the logarithmic operation is reversed by exponentiation.

Figure 9 shows two observations of the die, the estimated noise signal, and the object signal. It can be seen that ICA has successfully removed background speckle from the reconstruction of the die. A reduction of speckle can also be observed in the spots of the die. 


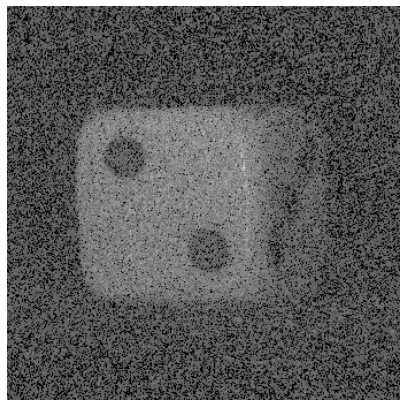

(a)

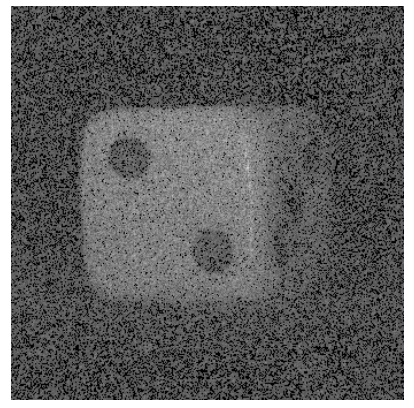

(b)

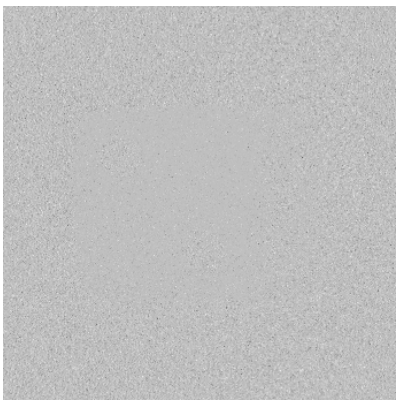

(c)

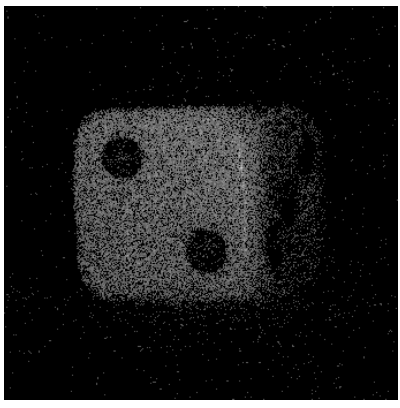

(d)

Figure 9. ICA applied to a hologram of a die. (a) and (b) are the two observations, (c) estimated noise source and (d) estimated object source.

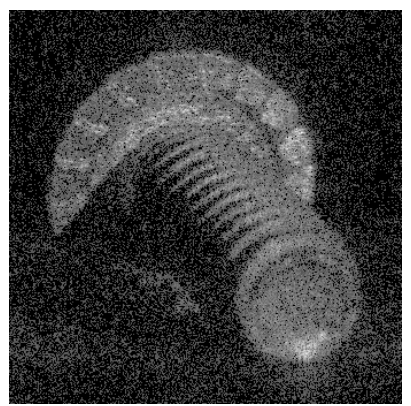

(a)

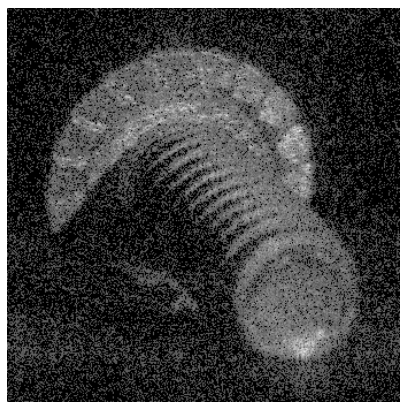

(b)

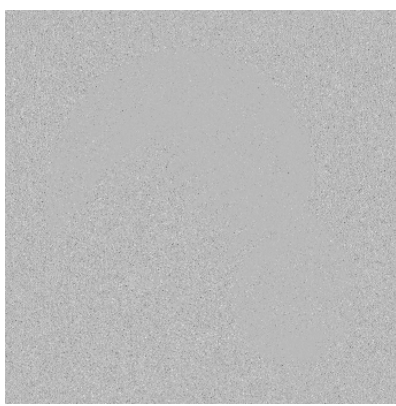

(c)

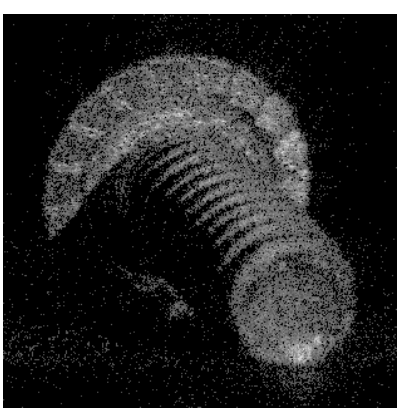

(d)

Figure 10. ICA applied to a hologram of a bolt. (a) and (b) are the two observations, (c) estimated noise source and (d) estimated object source.

The same experiment was repeated, this time using the bolt. Figure 10 shows two observations of the bolt, the estimated noise signal, and the object signal. Once again, it can be seen that ICA has successfully removed background speckle from the reconstruction of the bolt.

In Fig. 11 zoomed in $300 \times 300$ pixel regions of the reconstruction of the bolt and the estimated source signal that ICA obtains are shown. Since background speckle noise has been removed in the estimated ICA figure, the outline of the bolt is more visible.

It should be noted that the die and the bolt have very different characteristics. The die has a uniform surface across its faces, with distinct features, such as its spots, whereas the bolt is a much more textured object with a large degree of variance along it. Our technique has worked well in removing background speckle from both objects.

\section{EXTRACTION OF 3D SHAPE INFORMATION FROM A DIGITAL HOLOGRAMS}

In this section, we present a technique that converts a digital hologram into a surface in 3D space. We apply a technique called depth-from-defocus to our digital holograms to create depth maps of the objects encoded in our holograms.

\subsection{Focus Measures}

Focus measures are image assessment operations that attempt to characterize the degree of blurring exhibited in an image. Typical applications of such operations are in the areas of autofocus systems ${ }^{38,39}$ and $3 \mathrm{D}$ reconstruction systems. ${ }^{40}$ A good focus measure, when applied to the data should exhibit a robust well defined 


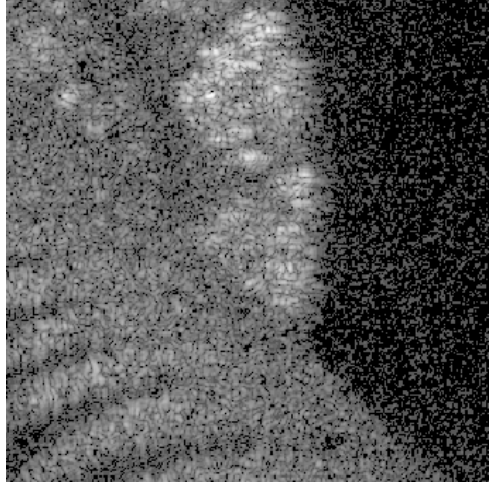

(a)

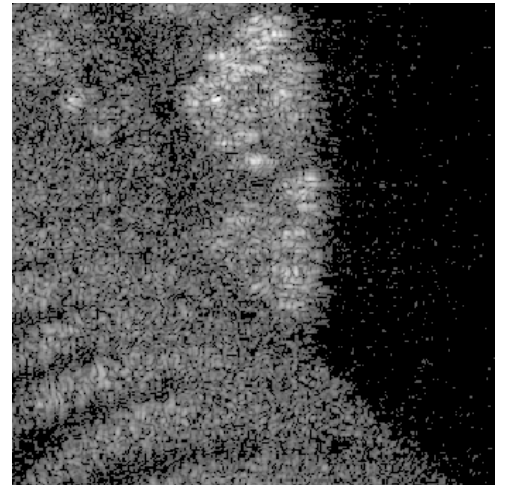

(b)

Figure 11. This shows a zoomed in region of the top right corner of the bolt. (a) one of the observations and (b) ICA estimated source signal.

characteristic such as an absolute maximum or an absolute minimum when the data is in focus. It should also be computationally efficient.

A digital hologram $H_{0}(x, y)$ contains sufficient amplitude and phase information to reconstruct the complex field $U(x, y, z)$ in a plane in the object beam at any distance $z$ from the camera. ${ }^{5,6,44}$ This can be calculated from the Fresnel approximation ${ }^{45}$ as

$$
U(x, y, z)=\frac{-\mathrm{i}}{\lambda z} \exp \left(\mathrm{i} \frac{2 \pi}{\lambda} z\right) H_{0}(x, y) \star \exp \left[\mathrm{i} \pi \frac{\left(x^{2}+y^{2}\right)}{\lambda z}\right],
$$

where $\lambda$ is the wavelength of the illumination and $\star$ denotes a convolution operation. At $z=d$, and ignoring errors in digital propagation due to discrete space (pixelation) and rounding, the discrete reconstruction $U(x, y, z)$ closely approximates the physical continuous field $U_{0}(x, y)$.

It is possible to reconstruct a hologram at unlimited depths by altering the distance parameter $z$. The most in-focus $2 \mathrm{D}$ reconstruction of this scene is taken by evaluating the focus measure at intervals over the range of $z$ values. This relies on the assumption that the large majority of the scene is in focus at a certain depth. If there are multiple objects at different distances the scene must be decomposed into blocks. Each block is processed using a focus measure to gather the depth information. This depth information can then be used to create a depth-map of the scene.

A number of approaches for the recovery of 3D information from digital holograms have been proposed in literature. Liebling ${ }^{38}$ uses a wavelet based technique to automatically set the distance parameter for numerical reconstruction of the complex wave field of a digital hologram. This results in the most in-focus reconstruction of the full complex wave field. Gillespie ${ }^{39}$ proposes the use of self-entropy of a hologram's quantized phase to calculate the sharpness of a numerically reconstructed complex wave field. This is calculated by,

$$
E=-\sum_{i=1}^{n} \sum_{j=1}^{n} P_{I(i, j)} \log P_{I(i, j)}
$$

where $P_{I(i, j)}$ is the probability of a pixel occurring in the input block $I$ of size $n$. Ma ${ }^{40}$ proposes the use of variance as a focus measure to gather $3 \mathrm{D}$ depth information from a digital hologram. This is defined as,

$$
V=\frac{1}{n \times n} \sum_{i=1}^{n} \sum_{j=1}^{n}[I(i, j)-\bar{I}]^{2}
$$

where $I$ is the input block and $\bar{I}$ is the mean of the input block. Through block processing of the complex wave field and application of the variance measure this approach has been successful in creating a 3D image of a digitized analog hologram. 


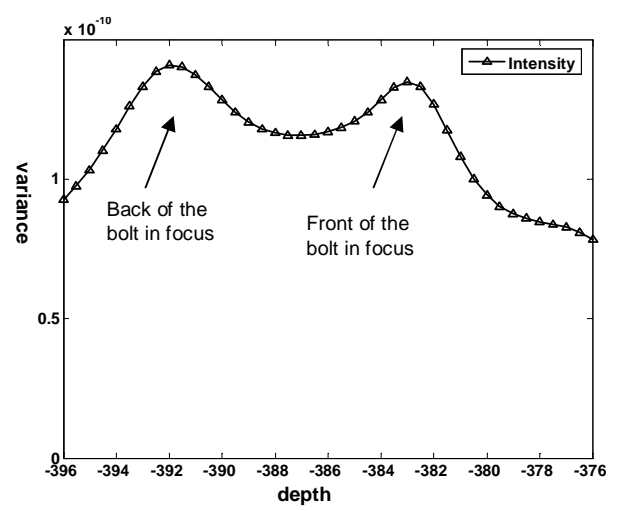

Figure 12. Plot of variance as applied to a full window of the bolt hologram.

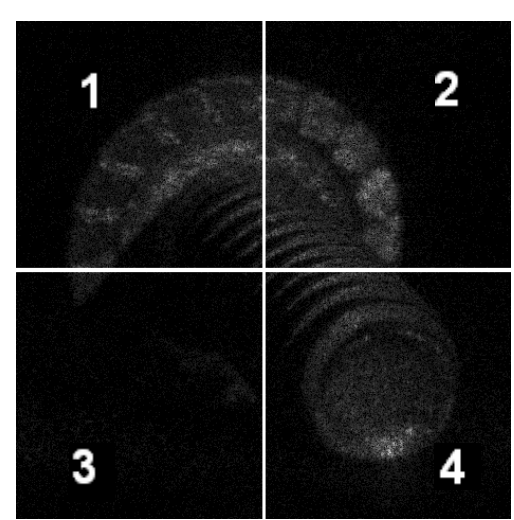

(a)

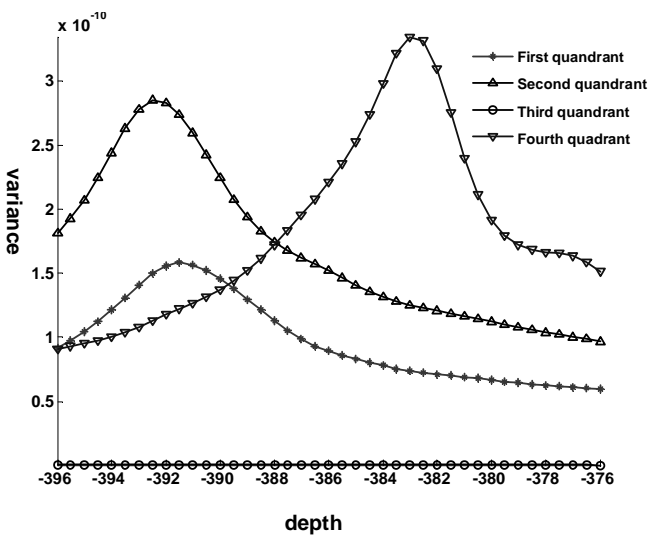

(b)

Figure 13. Bolt hologram a) split into four quadrants and b) variance plots for these quadrants.

Our holograms are reconstructed at a set of different depths $L$ using Equation 12 and starting at a depth $d_{0}$. The size of each reconstruction is $M \times N$ with $\Delta z$ being the interval between reconstructions.

$$
z_{e}=d_{o}-l \Delta z \quad l=1,2, \ldots, L
$$

The absolute depth for the experiments is described by $z_{e}$, equation 15 . We calculate the focus at each depth by separating each reconstruction into blocks of size $n \times n$, creating $m^{\prime} \times n^{\prime}$ blocks. Each of these blocks are processed using variance as a focus measure. The estimated depth for each block is evaluated by finding the one which exhibits a maximum. This gives us the 3D information we need in the form of a depth map, where each value in the depth map represents the distance from the hologram plane to the corresponding block in the scene.

\subsection{Application of variance as a focus measure to digital holograms}

To test the applicability of variance as a focus measure to our digital holograms we first attempted to focus a $1024 \times 1024$ window from a bolt hologram. One $n \times n$ block was taken as the input for the variance focus measure. The algorithm from Sect. 5.1 was used with forty different depth reconstructions, $\Delta z=0.5 \mathrm{~mm}$ and $d_{0}=-396$. Figure 12 shows the results of this experiment. It can be seen that the maximum variance occurs at $-392 \mathrm{~mm}$, where the back of the bolt is in focus. Variance also produces a local maximum at $-383 \mathrm{~mm}$, where the front of the bolt is in focus. Both amplitude and intensity exhibit similar properties with respect to variance. Either could be used, but we have chosen intensity for our experiments.

In subsequent experiments, we used smaller blocks within the $1024 \times 1024$ window of the hologram. The hologram was split into four equal quadrants of size $512 \times 512$, Fig. 13 (a). Each block was processed, and the depth which produced the maximum variance was taken as the estimated depth for the given block. Variance 


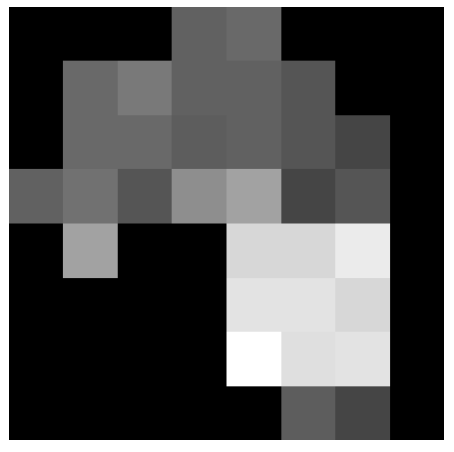

(a)

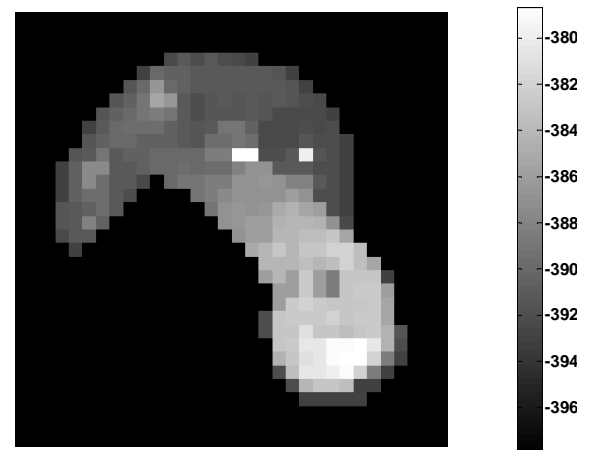

(b)

Figure 14. 2D depth map of the bolt hologram using variance with a) a $128 \times 128$ block size, b) a $32 \times 32$ block size.

gave us distinct maxima for three of the four quadrants. For quadrant 1 variance returns a maximum at depth $-391.5 \mathrm{~mm}$ and for quadrant 2 a maximum at depth $-392.5 \mathrm{~mm}$. Considering that the region of the back of the bolt visible in quadrant 1 is closer to the hologram plane than the region in quadrant 2, this was a positive result. Variance also correctly focuses quadrant 4, identifying $-383 \mathrm{~mm}$ as the depth. Quadrant 3 has very little object data in it, and contains shadows and background. For this input, variance returns a value three orders smaller than that for the other quadrants. We then set blocks whose variance is below this value to be background regions. We chose a threshold value of $10^{-13}$ for all experiments using variance.

We used a range of block sizes as inputs to variance. Figure 14 (a) shows the results of calculating variance on a $128 \times 128$ block size. The resulting depth map was of resolution $8 \times 8$. No median filtering was applied because it had a destructive affect on the depth map. In Fig. 14 (b) the 2D depth map obtained from calculating variance on a $32 \times 32$ block size is displayed. The depth map was thresholded, and this time median filtered was applied. The filtering neighborhood was of size $3 \times 3$. The main three regions that can be focused in the bolt are the front, the back and the threaded area. Variance seems to calculate the correct depths for these regions, and also identifies a range of depths in between them.

\section{CONCLUSION}

In this paper, we have detailed recent developments made by our group, in the areas of encryption, compression, noise removal using Independent Component Analysis (ICA), and a depth-from-defocus technique, applied to digital holograms of real world objects.

Our 3D objects are real-world objects captured using phase-shift interferometry (PSI). Encryption is performed with a phase mask in the Fresnel domain. We studied the sensitivity of the decoding system, to the finite phase quantization of the phase-only SLM used, to represent the encrypted image and the decoding mask.

We then used a Kohonen competitive neural network to compress our encrypted holographic data. We achieved reduced NRMS error and increased compression ratios using this nonuniform quantization technique. We found that as few as 2 bits in each real and imaginary value (corresponding to a compression ratio of 32 ) results in good quality decompressed and decrypted 3-D object reconstructions. Nonuniform quantization not only performs significant compression itself, it will also reduce the number of symbols (for Huffman) and introduce structure into the bit stream (for LZ77 and LZW) to allow them to perform further compression. Combined with quantization compression, the optical encryption technique based on phase-shift digital holography is suitable for a range of secure and efficient 3D object storage and transmission applications.

We have also successfully applied standard ICA to the removal of background speckle from digital holograms. To the best of our knowledge, we are the first group to attempt speckle noise reduction in digital holograms using ICA. Thus far, we have not been successful at improving the objects themselves in the scene. We have considered speckle noise to simply be a multiplicative noise, but the reality is that it is far more complicated 
than that. Future work will attempt to more closely model speckle as it occurs in nature, in an effort to achieve even better results. We will also look at ways to improve the estimated ICA signals by further image processing.

Finally, we presented a technique that converts a digital hologram into a surface in 3D space. Using variance as a focus measure we created a depth map which gave use the $3 \mathrm{D}$ information that was stored in our digital hologram. When the hologram was split into four quadrants, each quadrant was focused correctly using variance by taking an absolute maximum. Our holograms are $2048 \times 2048$ in size and the objects can be as small as $4 \mathrm{~mm}$. For this reason we need a focus measure capable of utilizing small block sizes to distinguish detail and produce an accurate depth map. Initial work in this area has shown variance can produce a high quality depth map.

Our work is part of a larger project to apply and adapt image processing algorithms and techniques to digital holographic data.

\section{Acknowledgements}

The authors wish to thank Enrique Tajahuerce and Yann Frauel for the use of their digital hologram data. This publication has emanated from research conducted with the financial support of Science Foundation Ireland, Enterprise Ireland, and the Embark Initiative of the Research Council for Science, Engineering, and Technology.

\section{REFERENCES}

1. J. W. Goodman and R. W. Lawerence, "Digital image formation from electronically detected holograms," Applied Physics Letters 11, pp. 777-778, Dec. 1967.

2. L. P. Yaroslavskii and N. S. Merzlyakov, Methods of Digital Holography, Consultants Bureau, New York, 1980. Translated from Russian by Dave Parsons.

3. J. H. Bruning, D. R. Herriott, J. E. Gallagher, D. P. Rosenfeld, A. D. White, and D. J. Brangaccio, "Digital wavefront measuring interferometer for testing optical surfaces and lenses," Applied Optics 13, pp. 26932703, Nov. 1974 .

4. U. Schnars and W. P. O. Jüptner, "Direct recording of holograms by a ccd target and numerical reconstruction," Applied Optics 33, pp. 179-181, Jan. 1994.

5. I. Yamaguchi and T. Zhang, "Phase-shifting digital holography," Opt. Lett. 22(16), pp. 1268-1270, 1997.

6. B. Javidi and E. Tajahuerce, "Three-dimensional object recognition by use of digital holography," Opt. Lett. 25(9), pp. 610-612, 2000.

7. B. Javidi and J. L. Horner, "Optical pattern recognition for validation and security verification," Optical Engineering 33, pp. 1752-1756, June 1994.

8. P. Réfrégier and B. Javidi, "Optical image encryption based on input plane and Fourier plane random encoding," Optics Letters 20, pp. 767-769, Apr. 1995.

9. L. Neto and Y. Sheng, "Optical implementation of image encryption using random phase encoding," Optical Engineering 35, 1996.

10. G. Unnikrishnan, J. Joseph, and K. Singh, "Optical encryption system that uses phase conjugation in a photorefractive crystal," Applied Optics 37, pp. 8181-8186, Dec. 1998.

11. O. Matoba and B. Javidi, "Encrypted optical memory system using three-dimensional keys in the Fresnel domain," Optics Letters 24, pp. 762-764, June 1999.

12. P. C. Mogensen and J. Glückstad, "Phase-only optical encryption," Optics Letters 25(8), pp. 566-568, 2000.

13. B. Javidi and T. Nomura, "Securing information by use of digital holography," Optics Letters 25, pp. 28-30, Jan. 2000.

14. S. Lai and M. A. Neifeld, "Digital wavefront reconstruction and its application to image encryption," Optics Communications 178, pp. 283-289, May 2000.

15. E. Tajahuerce, O. Matoba, S. C. Verrall, and B. Javidi, "Optoelectronic information encryption with phaseshifting interferometry," Applied Optics 39, pp. 2313-2320, May 2000.

16. E. Tajahuerce and B. Javidi, "Encrypting three-dimensional information with digital holography," Applied Optics 39, pp. 6595-6601, Dec. 2000.

17. B. M. Hennelly and J. T. Sheridan, "Optical image encryption by random shifting in fractional Fourier domains," Optics Letters 28, pp. 269-271, Feb. 2003. 
18. N. Nishchal, J. Joseph, and K. Singh, "Fully phase encryption using fractional Fourier transform," Optical Engineering 42, pp. 1583-1588, 2003.

19. J. Schwider, B. Burow, K. E. Elsner, J. Grzanna, and R. Spolaczyk, "Digital wavefront measuring interferometry: some systematic error sources," Applied Optics 22, pp. 3421-3432, 1983.

20. Y. Frauel, E. Tajahuerce, M.-A. Castro, and B. Javidi, "Distortion-tolerant three-dimensional object recognition with digital holography," Applied Optics 40, pp. 3887-3893, Aug. 2001.

21. J. J. G. Unnikrishnan and K. Singh, "Optical encryption by double-random phase encoding in the fractional Fourier domain," Optics Letters 25, pp. 887-889, 2000.

22. B. Hennelly and J. Sheridan, "Random phase and jigsaw encryption in the Fresnel domain," Optical Engineering 28, Oct. 2004.

23. T. J. Naughton and B. Javidi, "Compression of encrypted three-dimensional objects using digital holography," Optical Engineering 43, pp. 2233-2238, Oct. 2004.

24. G. Unnikrishnan, M. Pohit, and K. Singh, "A polarization encoded optical encryption system using ferroelectric spatial light modulator," Optics Communications 185, pp. 25-31, 2000.

25. B. Hennelly and J. Sheridan, "Fast numerical algorithm for the linear canonical transform," Journal of the Optical Society of America 22, May 2005.

26. J. Goodman and A. Silvestri, "Some effects of Fourier domain phase quantization," IBM J. Res. Develop. 14, pp. 478-484, 1970.

27. W. Dallas and A. Lohmann, "Phase quantization in holograms," Appl. Opt. 11, pp. 192-194, 1972.

28. T. Nomura, A. Okazaki, M. Kameda, Y. Morimoto, and B. Javidi, "Digital holographic data reconstruction with data compression," pp. 235-242.

29. T. Naughton, Y. Frauel, B. Javidi, and E. Tajahuerce, "Compression of digital holograms for threedimensional object reconstruction and recognition," Appl. Opt. 41(20), pp. 4124-4132, 2002.

30. O. Matoba, T. Naughton, Y. Frauel, N. Bertaux, and B. Javidi, "Real-time three-dimensional object reconstruction by use of a phase-encoded digital hologram," Appl. Opt. 41(29), pp. 6187-6192, 2002.

31. T. J. Naughton, J. B. McDonald, and B. Javidi, "Efficient compression of Fresnel fields for Internet transmission of three-dimensional images," Applied Optics 42, Aug. 2003.

32. B. Javidi, A. Sergent, G. Zhang, and L. Guibert, "Fault tolerance properties of a double phase encoding encryption technique," Optical Engineering 36, pp. 992-998, 1997.

33. F. Goudail, F. Bollaro, B. Javidi, and P. Réfrégier, "Influence of a perturbation in a double phase-encoding system," Journal of the Optical Society of America A 15, pp. 2629-2638, 1988.

34. T. J. Naughton and B. Javidi, "Encryption and decryption of three-dimensional objects using digital holography,"

35. M. Françon, Laser Speckle and Application in Optics, Acedemic, New York, 1979.

36. A. Hyvärinen, "Fast and robust fixed point algorithms for independent component analysis," IEEE Trans. on Neural Networks 10(3), pp. 626-634, 1999.

37. A. Hyvärinen, J. Karhunen, and E. Oja, Independent Component Analysis, Wiley, New York, 1991.

38. M. Liebling and M. Unser, "Autofocus for digital fresnel holograms by use of a fresnelet-sparsity criterion," Journal of the Optical Society of America A 21(12), pp. 2424-2430, 2004.

39. J. Gillespie and R. King, "The use of self-entropy as a focus measure in digital holography," Pattern Recognition Letters 9(1), pp. 19-25, 1989.

40. L. Ma, H. Wang, Y. Li, and H. Jin, "Numerical reconstruction of digital holograms for three-dimensional shape measurement," Journal of Optics A: Pure Applied Optics 6(4), pp. 396-400, 2004.

41. P. Comon, "Independent component analysis-a new concept?," Signal Processing 36, pp. 287-314, 1994. Special issue on Higher-Order Statistics.

42. "The FastICA MATLAB package.," 1994. Special issue on Higher-Order Statistics.

43. A. Hyvärinen and E. Oja, "Independent component analysis: Algorithms and applications," Neural Networks 13(4-5), pp. 411-430, 2000.

44. L. Onural and P. Scott, "Digital decoding of in-line holograms," Opt. Eng. 26(11), pp. 1124-1132, 1987.

45. J. Goodman, Introduction to Fourier Optics, Roberts and Company, Englewood, Colorado, third ed., 2005. 